\title{
HARMONIZAÇÃO DO SORRISO ATRAVÉS DA CIRURGIA PLÁSTICA PERIODONTAL: UM RELATO DE CASO
}

\author{
Smille harmonization using periodontal plastic surgery: a case report \\ Harmonización de la sonrisa através de la cirugía plastica periodontal: un relato de caso \\ Evandro Franco da Rocha1*, Barbara Hemmer ${ }^{1}$, Valéria Campanelli Franco da Rocha1.
}

\begin{abstract}
RESUMO
Objetivo: Descrever a harmonização do sorriso, através da cirurgia plástica gengival em uma condição descrita como sorriso gengival. Detalhamento do caso: Paciente, sexo feminino, 21 anos de idade, apresentou-se na Clínica de Odontologia da FURB, insatisfeita com a estética vermelha de seu sorriso. Realizados os exames periodontais, e comprovado o status de saúde periodontal, indicou-se e a cirúrgica plástica periodontal. Modelos prévios, através de um medidor de proporcionalidade de Chu, permitiram uma correta avaliação do aumento da coroa clínica dos dentes necessário e mantendo uma proporção harmônica dos mesmos. Este ensaio, após testado na paciente através de um mockup, serviu para a montagem do planejamento cirúrgico para diminuição da estética vermelha. $O$ controle fotográfico foi realizado antes do ato cirúrgico, no transcirúrgico, no pós operatório imediato, aos 7,14, 45, 60 e 90 dias após a realização da cirurgia. Os resultados permitiram obter um contorno gengival completamente estabelecido, arco côncavo gengival harmônico e ausência de discrepância nas curvaturas. Considerações finais: Concluiu-se que a realização da cirurgia plástica periodontal permitiu um resultado estético satisfatório com diminuição do sorriso gengival da paciente.
\end{abstract}

Palavras-chave: Sorriso, Gengiva, Mockup.

\begin{abstract}
Objective: To describe the harmonization of smile, through gingival plastic surgery in a condition described as gingival smile. Case detail: Patient, female, 21 years old, presented at the FURB Dentistry Clinic, unsatisfied with the red aesthetics of his smile. Periodontal exams were performed, and the periodontal health status was confirmed, and the periodontal plastic surgery was indicated. Previous models, through a proportionality meter of $\mathrm{Chu}$, allowed a correct evaluation of the increase of the clinical crown of the necessary teeth, and maintaining a harmonious proportion of them. This assay, after being tested in the patient through a mockup, served for the assembly of the surgical planning decrease of the red aesthetics. Photographic control was performed before surgery, in the surgery, in the immediate postoperative period, at 7 and at 14 , 45, 60 and 90 days after surgery. Results: The results allowed to obtain a completely established gingival contour, concave gingival arc harmonic and absence of discrepancy in the curvatures. Final considerations: It was concluded that the accomplishment of periodontal plastic surgery allowed a satisfactory aesthetic result with reduction of the patient's gingival smile.
\end{abstract}

Key words: Smile, Gingiva, Mockup.

1Universidade Regional de Blumenau (FURB), Blumenau-SC *E-mail: efrocha@furb.br

SUBMETIDO EM: 4/2019 | ACEITO EM: 5/2019 | PUBLICADO EM: 7/2019 


\section{RESUMEN}

Objetivo: Describir la armonización de la sonrisa, a través de la cirugía plástica gingival en una condición descrita como sonrisa gingival. Detalle del caso: Paciente, sexo femenino, 21 años de edad, se presentó en la Clínica de Odontología de la FURB, insatisfecha con la estética roja de su sonrisa. Se realizaron los exámenes periodontales, y comprobado el estado de salud periodontal, se indicó la cirugía plástica periodontal. Los modelos previos, a través de un medidor de proporcionalidad de Chu, permitieron una correcta evaluación del aumento de la corona clínica manteniendo una proporción armónica de los mismos. Este ensayo, después de probado en la paciente a través de un mockup, sirvió para el montaje de la planificación quirúrgica disminución de la estética roja. El control fotográfico fue realizado antes del acto quirúrgico, en el transcirúrgico, en el postoperatorio inmediato, a los 7 días, ya los 14, 45, 60 y 90 días después de la realización de la cirugía. Obtuvimos un arco cóncavo gingival armónico y ausencia de discrepancia en las curvaturas. Consideraciones finales: Se concluyó que la realización de la cirugía plástica periodontal permitió un resultado estético satisfactorio con disminución de la sonrisa gingival de la paciente.

Palabras clave: Sonrisa, Goma, Mockup.

\section{INTRODUÇÃO}

A busca pela excelência estética não é um parâmetro atual, ela ocorre desde tempos remotos. Entretanto o conceito de beleza é subjetivo pois dependente de quem o analisa, o qual sofre influencias do meio cultura e social em que vive, tornando este conceito subjetivo (PINTO TB, 2016).

A sociedade tem procurado cada vez mais os padrões de beleza odontológica. Como fundamento básico temos a harmonização dental, não apenas pela cor dos elementos dentais, mas também na relação de proporção entre os dentes e altura cervical das margens gengivais. (LIMA APM, et al., 2016).

O sorriso tem um papel marcante na expressão facial, ele que revela estados de satisfação, alegria e felicidade que são contribuintes ao bem-estar e autoconfiança do indivíduo (PINTO TB, 2016).

O sorriso gengival é diagnosticado através das classificações da linha do sorriso, linha imaginária que acompanha a borda inferior do lábio superior ao sorrir (BORGHETTI A, 2010). Este parâmetro corresponde verticalmente a quantidade de estrutura dental visível durante o sorriso (PINTO TB, 2016).

Segundo Tjan AHL, et al. (1984), as linhas do sorriso são classificadas em três categorias: a primeira categoria foi denominada linha do sorriso alta, quando mostra toda extensão coronária dos dentes anteriores superiores juntamente com uma faixa de tecido gengival, encontrando-se exposições gengivais acima de $3 \mathrm{~mm}$, caracterizando o sorriso gengival. A segunda categoria denominou-se de linha do sorriso média, quando de 75 a 100\% dos dentes anteriores superiores juntamente com a gengiva interproximal é mostrada. $\mathrm{Na}$ terceira categoria denominada linha do sorriso baixa, menos de $75 \%$ dos dentes anteriores superiores aparece.

Segundo Borghetti A (2010) e Faria GC et al., (2015), a predominância de sorriso gengival em adultos jovens é de aproximadamente $10 \%$, sendo essa condição mais frequente em mulheres do que nos homens, e tende a regredir gradualmente com a idade, em decorrência do surgimento de flacidez nos lábios superiores e inferiores, levando a uma diminuição da exposição dos incisivos superiores e um aumento da exposição dos incisivos inferiores. Para Ribeiro FS, et al., (2004), o sorriso gengival pode ser descrito como uma anomalia de desenvolvimento, caracterizado pela exposição excessiva de gengiva quando o paciente sorrir e Fernandes TV, et al., (2014), citam que existem diversas etiologias e opções de tratamento para a correção do sorriso gengival, sendo para alguns casos o diagnóstico de tratamento multidisciplinar.

Saba-Chujfi, E. e Santos-Pereira, S. A. (2007), Rivero GVM e Silva GCH (2006), Santos GG e Rego DM. (2007) e Pires CV et al (2010) argumentam que o profissional especialista em periodontia é o responsável pelo diagnóstico e tratamento do aumento gengival associado a erupção passiva e havendo a presença de 
hiperatividade labial associada, é necessária a atuação de uma equipe multidisciplinar composta pelo periodontista, dermatologista e cirurgião plástico. Com a técnica da cirurgia plástica periodontal permite-se a exposição da coroa anatômica, o aumento da coroa clínica e a maximização da aparência do sorriso, tornando-o mais estético (MENDES APM, 2011). Para a realização da cirurgia deve-se levar em conta três condições: (1) localização da crista óssea alveolar em relação à junção amelocementária de aproximadamente, 1,5 a $2 \mathrm{~mm}$, (2) espessura apropriada de osso alveolar e (3) largura de gengiva queratinizada. Caso não se observem estas condições, podem ocorrer recidivas (PINTO TB, 2016).

Zanatta FB et al., (2010), concluíram que o meio mais adequado para diagnosticar a localização da crista óssea alveolar em relação à junção amelocementária é através da sondagem transperiodontal.

Majzoub ZAK et al., (2014), sugerem que o posicionamento apical do tecido gengival pode ser determinado com o auxílio de um guia cirúrgico em resina acrílica (mockup), o qual funciona como um orientador para o recontorno dos tecidos moles e duros durante a cirurgia, o mesmo tem como vantagem demonstrar uma prévia do resultado final do tratamento ao paciente e ao cirurgião dentista.

Segundo Trevisani RS e Meusel DRD (2014), confeccionar o mockup é extremamente importante pois auxilia o planejamento e proporciona uma relação adequada de proporcionalidade entre estes e os lábios e poderá ser utilizado como uma guia cirúrgica. Além disso a utilização do mockup como guia cirúrgica diminui o tempo cirúrgico além de aumentar a precisão e previsão do resultado final do procedimento, podendo ser obtido através de uma guia de silicone ou resina sobre um modelo de gesso obtido previamente onde já se realizou o enceramento para aumento dos dentes (PIRES CV et al., 2010 E PAIVA AO et al., 2013).

Para a determinação dos valores médios de comprimento e largura dos dentes maxilares anteriores pode ser utilizado como auxiliar o calibrador ou sonda de Chu. Os medidores estéticos da Chu são uma série de medidores codificados por cores, com design inovador e fornecem uma abordagem passo a passo, baseada na biologia, para o alongamento da coroa estética periodontal. Este instrumento tem formato semelhante a uma cruz e em cada uma de suas extremidades existem três marcações coloridas. Estas marcações se relacionam a altura e largura do elemento dental de forma que este possa adquirir uma proporção estética. Cada marcação deve se corresponder com outra da mesma cor. Desta forma o comprimento clínico ideal da coroa de um dente será determinado com base em relações estabelecidas de proporção dentária com as mesmas grandezas da proporção áurea, assim as larguras das marcações coloridas no braço horizontal são $75 \%$ a $80 \%$ dos comprimentos das marcações coloridas correspondentes no braço vertical (FLETCHER P. 2011; PINTO TB, 2016; NAUTIYAL A et al. 2016).

Este trabalho teve como objetivo relatar um caso clínico de uma paciente com sorriso gengival, na qual realizou-se a cirurgia plástica periodontal com o auxílio do mockup para a harmonização do sorriso e obtenção de previsibilidade do caso.

\section{DETALHAMENTO DO CASO}

Paciente de 21 anos, do sexo feminino, procurou a clínica de Odontologia da FURB, queixando-se de fator estético ao sorrir, relatando que está insatisfeita com a estética vermelha de seu sorriso, o qual está caracterizado por uma exposição gengival maior que $3 \mathrm{~mm}$ (Figura 4). A paciente durante a anamnese não relatou nenhuma alteração sistêmica de saúde, ao exame clínico pode-se verificar um excelente controle do biofilme dental, profundidade de sondagem periodontal menor ou igual a $2 \mathrm{~mm}$ com ausência de sangramento, ausência de doença periodontal, ausência de inflamação gengival, gengiva caracterizada por uma cor rosa pálido de superfície fosca e pontilhada.

Após criteriosa análise do sorriso, através de exame clínico e registro fotográfico, foram identificados como fatores etiológicos lábio superior hiperativo, erupção passiva alterada e crescimento maxilar vertical. $O$ tratamento de escolha para o caso foi cirurgia plástica periodontal com reposicionamento da margem gengival no sextante superior anterior, englobando dos dentes 13 ao 23 , utilizando o auxílio de um guia cirúrgico (mockup).

REAS/EJCH | Vol. Sup.26 | e780 | DOI: https://doi.org/10.25248/reas.e780.2019 Página 3 de 8 
Para a confecção do guia cirúrgico, necessitou de uma moldagem com um material de boa estabilidade dimensional e de presa rápida, (silicone de adição pesado, Variotime Kulzer®), para uma cópia detalhada de dentes e gengiva.

O modelo foi confeccionado com gesso especial Durone ${ }^{\circledR}$. Aplicaram-se sobre o modelo os princípios estéticos de zênites, bem como fez-se o uso da régua de Chu para delimitação do máximo de gengiva a ser removido. Realizou- se o enceramento das faces vestibulares até a área cervical demarcada.

O enceramento teve espessura aproximada de $2 \mathrm{~mm}$ e o término cervical foi biselado em relação a superfície do modelo de gesso. Após enceramento foi realizada uma moldagem com silicone de condensação Variotime Kulzer® sobre o mesmo para a obtenção do mockup. Com o mockup devidamente recortado e ajustado, acrescentou-se resina bisacrilica cor A1 Yprov Bisacrilß da YLLER para a obtenção do guia cirúrgico e para demonstração do caso para a paciente.

Antes de realizar a cirurgia periodontal, a paciente foi informada sobre os procedimentos a serem realizados, e através do uso do mockup posicionado em boca ela pode observar uma demonstração prévia do resultado final cirúrgico. Após essa demonstração, concordou com o tratamento.

O tratamento cirúrgico foi iniciado após a preparação da paciente com antissepsia intra e extra bucal, e com anestesia por bloqueio do infra orbital dos lados direito e esquerdo. Devido a sensibilidade durante o ato operatório foi necessário realizar anestesias infiltrativas palatinas como complemento. Em sequência foi realizada a sondagem para identificar a posição da junção cemento esmalte.

Após a sondagem posicionou-se o guia cirúrgico (mockup) em boca e em seguida foram feitas as demarcações dos pontos sangrantes com a sonda milimetrada Carolina do Norte HuFriedy® para a determinação do local exato da incisão em bisel interno.

Em sequência, fez-se a união dos pontos sangrantes do hemiarco direito com lamina de bisturi no $15 \mathrm{C}$ Lamedid $\AA$ e cabo de bisturi $n^{\circ} 3$ quinelato $\AA$, seguido de incisões submarginais, que juntos formaram um "colarinho" gengival (Figura 1), o qual foi removido logo em seguida.

Figura 1 - Sequência clínica da união dos pontos sangrantes.

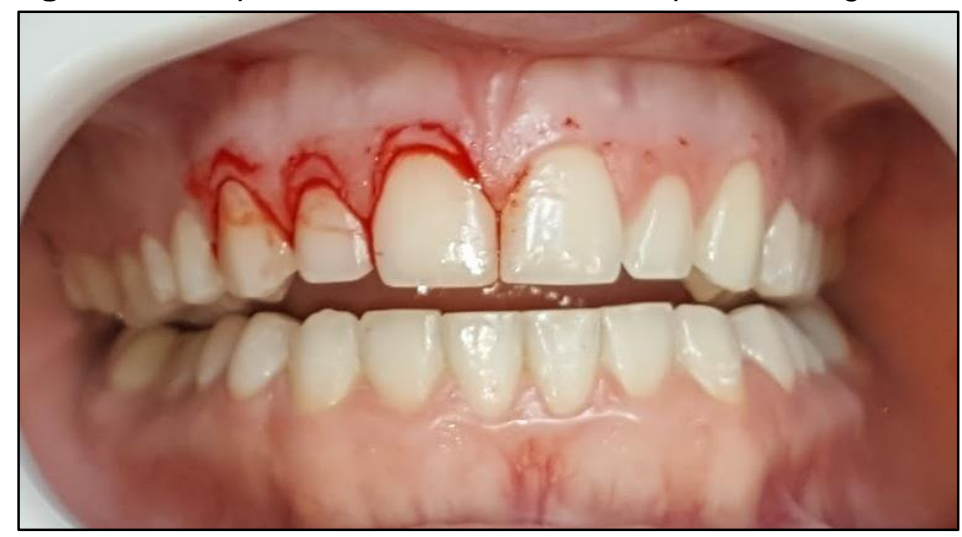

Fonte: Autores, 2019.

O refinamento do tecido gengival foi obtido com tesoura Goldman Fox Golgran Millennium $\AA_{\text {. }}$ O mesmo processo realizado no hemiarco direito foi repetido no hemiarco esquerdo. Após o refinamento gengival realizou-se a sondagem transperiodontal para a verificação das medidas das distâncias biológicas, que indicaram medidas inferiores a $3 \mathrm{~mm}$ da margem gengival até a crista óssea, indicando ausência do espaço biológico adequado. Posteriormente rebateu-se o retalho para regularização da margem óssea, e conformação dos sulcos, através de osteotomia, que se realizou com micro cinzel de Oschsenben ${ }^{\circledR}, \mathrm{n}^{\circ} 1,2 \mathrm{e}$

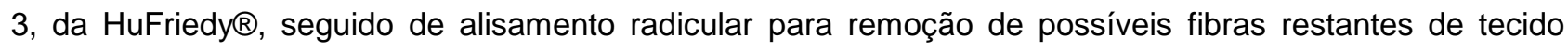
conjuntivo. Durante a cirurgia foi realizada irrigação com soro fisiológico. 
Para a finalização, o retalho foi reposicionado e suturado com fio reabsorvível vicryl 5.0 johnson\&Johnson® com pontos simples (Figura 2). Para que a paciente conseguisse higienizar normalmente seus dentes, optouse por não fazer o uso do cimento cirúrgico.

Figura 2 - Aspecto imediato após a realização cirúrgica.

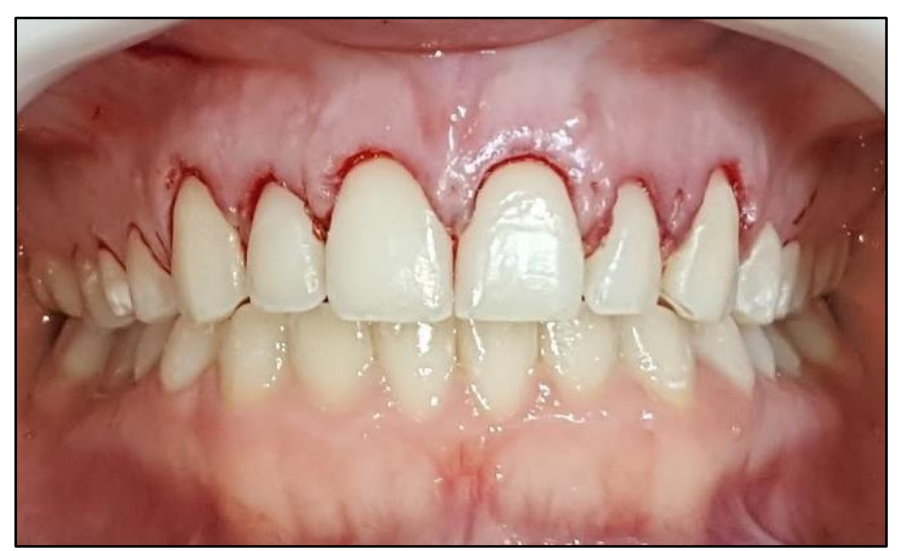

Fonte: Autores, 2019.

Para um melhor conforto pós-operatório e para o auxílio de cicatrização tecidual, foi feito uma aplicação de laser de baixa potência, marca duo MMOptics $\AA^{\circledR}$, na região, sendo colocado conforme o protocolo recomendado pelo fabricante: em 1 ponto cervical por elemento, em uma única aplicação durante 30 segundos em cada dente, com comprimento de onda 660nm (laser infravermelho), 3j (joules). (NUÑES SC, 2017).

Pela paciente não apresentar nenhuma contraindicação medicamentosa, prescreveu-se anti-inflamatório Nimesulida 100mg de 12 em 12 horas durante 03 dias associado ao anti-inflamatório ibuprofeno $600 \mathrm{mg}$ de 08 em 08 horas durante 03 dias. A remoção da sutura ocorreu 07 dias após a cirurgia (Figura 3), e os acompanhamentos ocorreram aos 07 (Figura 3), 14, 45, 60 (Figura 3) e 90 dias (Figura 5).

Figura 3 - Aspecto clínico após 7 e 60 dias.
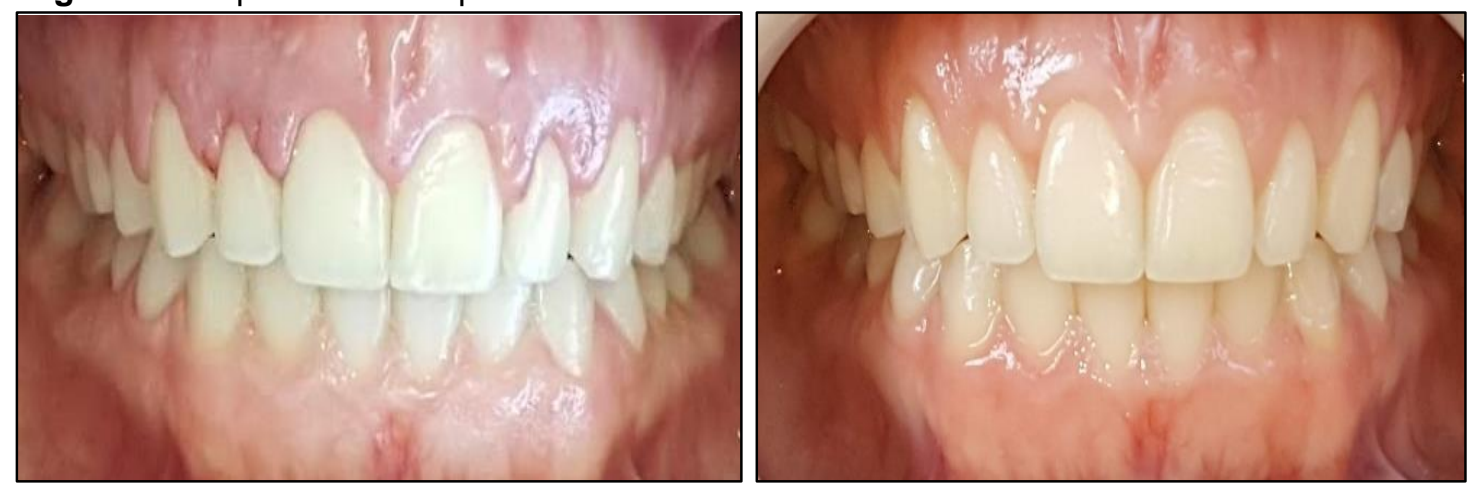

Fonte: Autores, 2019.

A cirurgia foi realizada apenas nos dentes anteriores, o que não comprometeu as distâncias biológicas dos dentes posteriores, nem alterou a estética do corredor bucal.

O resultado desse caso clínico, no pós-operatório imediato, atingiu praticamente uma réplica fiel do que foi proposto com o mockup. Observou-se uma correção na posição do zênite gengival, bem como o aumento das coroas clinicas, como o exibido pela avaliação estética. 
Houve uma diminuição no ganho de altura das coroas, após 7 dias, por conta da presença de edema, principalmente pela sua evidenciação nas papilas. $O$ aspecto é de bastante edema, neste período ainda não se retrata o resultado final esperado. Em uma visão lateral nota-se o arredondamento das papilas, que perderam o aspecto afilado por conta do edema.

Ao se comparar os resultados dos 7 aos 14 dias, observou-se uma redução do edema e um aumento das coroas clínicas, sendo nesse período a posição da gengiva marginal mais próxima ao que buscamos através do mockup cirúrgico, porém ainda o contorno gengival se encontra irregular, principalmente nos incisivos laterais.

Observou-se, aos 45 dias, um reposicionamento do zênite, bem próximo da junção cemento-esmalte, obtendo então o ganho que traçamos com o planejamento, nesse período ainda se observa um contorno gengival não bem definido, principalmente nos dentes 13 e 12 e no 22, as papilas ainda não possuem um aspecto afilado, e a margem gengival do 13 e 12 ainda apresentam eritema.

Com a cicatrização já completamente definida, aos 60 dias, as fibras já se encontravam reorganizadas, a ceratinização da margem gengival está completa, o aspecto de edema desapareceu, a cor da gengiva próxima ao natural e houve o aumento esperado das coroas clínicas.

No período de 90 dias observou-se um resultado similar aos dos 60 dias, com contorno gengival completamente estabelecido, com o arco côncavo gengival harmônico, com ausência de discrepância nas curvaturas e com aspecto tecidual de completa maturação, ausência de eritema marginal e papilar.

As papilas encontravam-se afiladas, o tecido gengival de coloração rosa claro, podendo afirmar- se que a cicatrização havia sido completada (Figura 4 e Figura 5).

Figura 4 - Sorriso inicial.

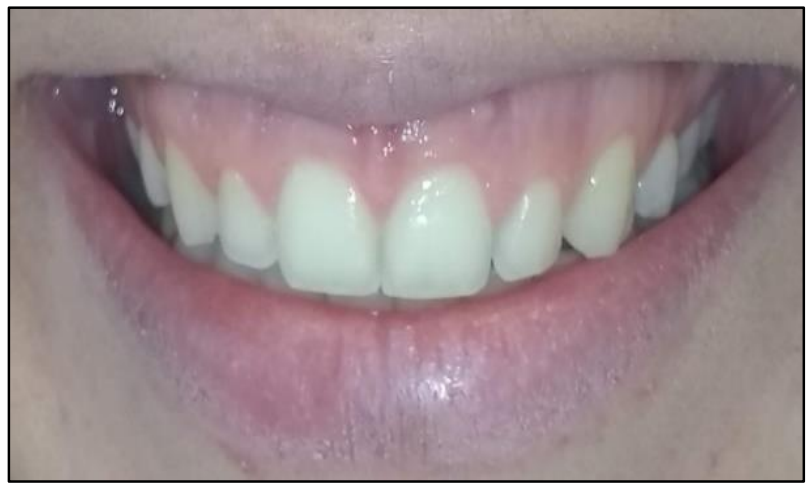

Fonte: Autores, 2019.

Figura 5 - Sorriso após 90 dias.

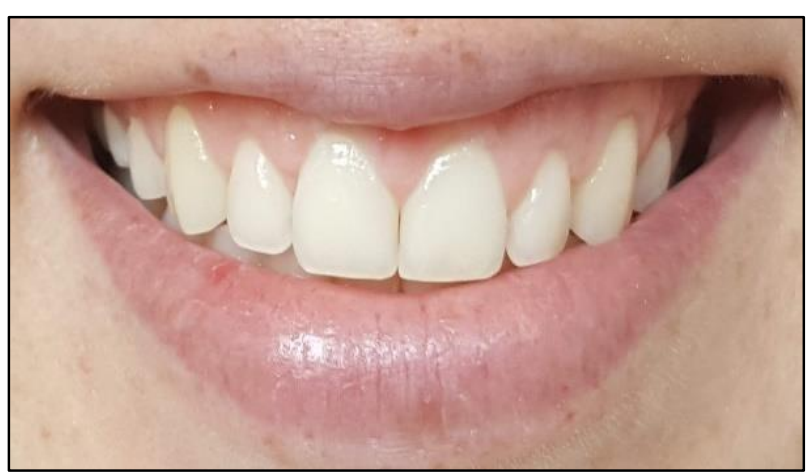

Fonte: Autores, 2019. 


\section{DISCUSSÃO}

Mestrener SR e Komatsu J (2002) descreveram que uma pessoa apresentando a linha do sorriso desviada, pode desenvolver um perfil psicológico alterado, com dificuldade de se relacionar e timidez excessiva. Adicionalmente Lima APM (2016) afirmou que quando o paciente se queixa da estética, por apresentar uma gengiva muito exposta, ou com formato irregular, mesmo que saudável, a mesma deve ser tratada para reestabelecer harmonia e continuidade da margem gengival. Neste caso a paciente selecionada apresentava uma faixa continua de gengiva maior que $3 \mathrm{~mm}$ durante o sorriso, e como no caso apresentado por Lima APM (2016) foi diagnosticada a presença de sorriso gengival causada pela erupção passiva alterada.

Exatamente por conta deste diagnóstico, Tjan AHL (1984) classificam esta linha do sorriso como "alta". Tudo levando a paciente a uma insatisfação estética e inibição ao sorrir.

Como Lima APM (2016), optamos pela correção harmônica do sorriso utilizando a cirurgia plástica gengival: cirurgia de gengivectomia com bisel interno, com associação de retalho para osteotomia e osteoplastia, porém levando em conta que antes de se dar início a qualquer tratamento odontológico o periodonto precisa estar saudável (BEZ CN, 2014), fez-se o exame clinico para identificação de algum sinal de doença periodontal e nada foi encontrado, assim, após o diagnóstico procedemos com a realização do planejamento.

Fernandes TV et al. (2014) e Bez CN (2014) defendem que em diversas situações clínicas, onde há perda de função e comprometimento estético, dental e gengival, o reestabelecimento se dá através de uma abordagem multidisciplinar, com inter-relação entre as técnicas restauradoras e técnicas cirúrgicas. Nesta multidisciplinariedade se encaixa o planejamento reverso através do mockup.

Feito desta forma, segundo Castro PHDF et al. (2010), está indicado para a cirurgia de correção de sorriso gengival, uma vez que oferece ao profissional segurança no ato cirúrgico, e confere ao procedimento alto grau de precisão e previsibilidade, bem como a possível visualização do profissional ao caso.

No planejamento do enceramento de diagnóstico deste caso clinico, levou-se em conta os valores médios de comprimento e largura dos dentes maxilares anteriores utilizando a régua de Chu, que possui três bandas coloridas.

As bandas vermelhas correspondem aos incisivos centrais, as bandas amarelas aos caninos e as bandas azuis aos incisivos laterais (PINTO TB, 2016) as quais respeitam a harmonia entre altura e largura das coroas clínicas dos dentes.

Além disso a efetivação do aumento das coroas dos dentes anteriores do nosso caso clínico, respeitou as condições do espaço biológico, em concordância com a literatura, a exemplo de Bez CN (2014) e Pinto TB (2016). Levando-se em conta os três princípios para a realização da cirurgia periodontal, segundo Pinto TB (2016): Localização da crista óssea alveolar em relação a junção amelo cementaria, sendo possível ser localizada através da sondagem transperiodontal/transcirurgica; Espessura do osso alveolar; Largura da gengiva queratinizada.

Na ausência de outro método disponível, optou-se por realizar a avaliação do espaço biológico através da sondagem transperiodontal. Festugatto FV et al., (2000), afirmaram que a sondagem transperiodontal é um método para a verificação do espaço biológico que produz medidas confiáveis concluindo que proporcionou $52,83 \%$ de fidelidade enquanto o método radiográfico periapical obteve $35,84 \%$ de fidelidade.

Tendo obtido medidas que denunciavam a invasão do espaço biológico após o aumento das coroas pela gengivectomia, o rebatimento do retalho cirúrgico foi obrigatório, para a realização da osteotomia e osteoplastia e reestabelecimento do espaço biológico ideal, em sintonia com Rigueira I, (1986) e Pires CV et al. (2010), obtendo-se um resultado estético satisfatório, com diminuição da exposição gengival proporcionando uma harmonia entre os tecidos gengivais e dentários, com preservação de função dental e saúde periodontal, concluindo-se que planejamentos prévios, através de mockup, proporcionam maior previsibilidade do resultado e maior satisfação da paciente após a realização cirúrgica. 


\section{REFERÊNCIAS}

1. BEZ CN Cirurgia periodontal em restaurações estéticas, Trabalho de conclusão de Curso (Graduação em Odontologia) Universidade Federal de Santa Catarina, Florianópolis, 2014; 61p.

2. BORGUETTI A. Cirurgia plástica periodontal. $2^{\mathrm{a}}$ Ed.Porto Alegre: Artmed, 2010. 464p.

3. BRAGA MS. et al. Cirurgia plástica periodontal para correção de erupção passiva alterada. Revista Periodontia, 2015; 25(4):64-68.

4. CASTRO PHDF. et al. Planejamento reverso na correção do sorriso gengival. Revista Periodontia, 2010, 20(3):42-46.

5. FARIA GJ. et al. A importância do planejamento multidisciplinar para correção do sorriso gengival: relato de caso clínico. Revista da Faculdade de Odontologia de Lins, 2015; 25(1):61-65.

6. FERNANDES TV et al. Abordagem multidisciplinar na reabilitação estética anterior. Revista APCD de Estética, 2014; 2(4):400-417.

7. FESTUGATTO FE, et al. Aumento de coroa clínica: comparação de técnicas de diagnóstico de invasão do espaco biológico do periodonto. Revista Periodontia, 2000; 9(1):42-49.

8. FLETCHER P. Biologic rationale of esthetic crown lengthening using innovative proportion gauges. International Journal of Periodontics and Restorative Dentistry, 2011; 31(5):523-532.

9. LIMA APM. Diferentes planejamentos e técnicas cirúrgicas para a correção do sorriso gengival: relato de caso. Trabalho de conclusão de curso (Graduação em Odontologia), Universidade de Brasília, Brasília, 2016, 48p.

10. MAJZOUB ZAK, et al. Crown lengthening procedures: A literature Review. Seminars in Orthodontics, 2014; 20:188-207.

11. MENDES APM. Sorriso gengival: etiologia, diagnóstico e opções de tratamento. Dissertação (Mestrado ntegrado em Odontologia), Universidade de Lisboa, Lisboa, 2011, 38p.

12. MESTRENER SR, KOMATSU J. Recuperação da linha do sorriso utilizando procedimentos cirúrgico e restaurador. Jornal Brasileiro de Dentística \& Estética, 2002; 1(3):226-230.

13. NAUTIYAL A, et al. Aesthetic Crown Lengthening Using Chu Aesthetic Gauges And Evaluation of Biologic Width Healing. Journal of Clinical and Diagnostic Research, 2016; 10(1):ZC51-ZC55.

14. NÚÑES SC. Protocolo de laserterapia e terapia fotodinâmica laser Duo. São Carlos: MMOptics, 2017, 51p.

15. PAIVA AO, et al. Enceramento diagnóstico e mock-up no planejamento das correções de sorrisos gengivais. Perionews, 2013; 7(5):453-459.

16. PINTO TB. Técnicas de correção do sorriso gengival. Dissertação (Mestrado Integrado em Odontologia), Universidade de Lisboa, Lisboa, 2016, 48p.

17. PIRES CV, et al. Procedimentos plásticos periodontais em paciente com sorriso gingival: relato de caso. Revista Periodontia, 2010; 20(1):48-53.

18. RIBEIRO FS et al. Surgical management of gummy smile caused by delayed passive eruption: A case report. Journal of Indian Academy of Aesthetic and Cosmetic Dentistry, 2004; 29:19-25.

19. RIGUEIRA I. Gengivectomia e gengivoplastia. In: Fundamentos de periodontia: (morfologia, patologia, prevenção e terapeutica aplicadas a clinica. Rio de Janeiro: Quintessence, 1986, 257p.

20. RIVERO GVM, SILVA GCH. Considerações estéticas na finalização ortodôntica em relação a linha do sorriso, contorno gengival e proporção dentária. Revista da Sociedade Brasileira de Ortodontia, 2006; 5(3):205-16.

21. SABA-CHUJFI E, SANTOS PSA. Periodontia: integração e resultados. São Paulo: Artes Médicas; 2007, 316p.

22. SANTOS GG, REGO DM. The influence of a gummy smile on lip seal. Journal of the International Academy of Periodontology, 2007; 9(2):53-7.

23. SILVA CMCLN. et al. Periodontia, estética orofacial e o tratamento multidisciplinar do sorriso gengival: relato de caso. Revista FIMCA, 2017; 4(1):72-80.

24. SOUZA LT. Sorriso gengival: relato de caso clínico. (Especialização em Periodontia), Escola De Medicina e Saúde Pública, Salvador, 2002, 20p.

25. TJAN AHL Some esthetic factors in a smile. et al. Journal of Prosthetic Dentistry, 1984; 51(1):24-28.

26. TREVISANI RS, MEUSEL DRD. Aumento de coroa clínica em dentes anteriores: relato de caso clinico. Journal of Oral Investions, 2014; 3(2):19-24.

27. ZANATTA FB et al. Comparison of different methods involved in the planning of clinical crown lengthening surgery. Brazilian Oral Research, 2010; 24(4):443-448. 